\title{
Bordetella bronchiseptica infection in stage IV Hodgkin's disease
}

\author{
DAVID B. Stoll* \\ M.D. \\ Shelia A. MurPheY† \\ M.D. \\ SAMir K. BALlas* \\ M.D. \\ *Cardeza Foundation for Hematologic Research, and $\dagger$ Department of Medicine, Division of \\ Infectious Diseases, Jefferson Medical College, Philadelphia, PA
}

\begin{abstract}
Summary
It is well known that patients with lymphoproliferative disorders have an altered immune status and are susceptible to opportunistic and non-opportunistic infections. The authors report the first case of a patient with advanced Hodgkin's disease who developed pneumonia caused by the animal pathogen Bordetella bronchiseptica. The most likely source of this organism was the patient's dog. While $B$. bronchiseptica is often responsible for outbreaks of pneumonia and septicaemia in wild and domestic animals, its role and incidence in human infections is not known. It is felt that patients with underlying lymphoproliferative diseases and pneumonia should be questioned about any contact with animals.
\end{abstract}

\section{Introduction}

Patients with lymphoproliferative diseases have an altered immune response and are susceptible to a variety of both non-opportunistic and opportunistic infections. The purpose of this paper is to report a patient with stage IV Hodgkin's disease, who developed pneumonia caused by Bordetella bronchiseptica (usually an animal pathogen) and Staphylococcus aureus. There is only one case in the literature of well documented fatal pneumonia due to $B$. bronchiseptica and that was in a malnourished and debilitated alcoholic (Gosh and Tranter, 1979). Another reported case describes B. bronchiseptica and pneumococcal septicaemia in a patient with Evans's syndrome who was treated with splenectomy and steroids (Gardner et al., 1970). No source for the pathogen was established in either case. In the present patient, the source of the organism was thought to be the patient's dog.

\section{Case report}

A 26-year-old female was admitted to Thomas Jefferson University Hospital (TJUH) in April of
1979 with fever and shortness of breath. Twelve years before admission she was diagnosed as having stage I Hodgkin's disease. She was treated with radiotherapy to the affected area. After 5 years of symptom-free disease, stage IV Hodgkin's disease was diagnosed when she developed 2 pulmonary nodules and bilateral pleural effusions. She was treated cyclically with vincristine, prednisone and procarbazine. Clinical remission was achieved. She was followed-up as an out-patient and was given occasional cycles of chemotherapy on an empirical basis.

Twelve weeks before her admission to TJUH, the patient complained of shortness of breath and a chest X-ray showed that a right apical pneumothorax had developed. This slowly progressed, necessitating admission to (another) hospital and the placement of a chest tube 11 weeks later. She developed fever of $39.4^{\circ} \mathrm{C}$ and a severe productive cough 4 days after this. Chest X-ray showed diffuse bilateral pulmonary infiltrates. She was transferred to TJUH for respiratory intensive care after 9 days in hospital. Past history revealed that a sick pet dog had died 5 days before her admission to hospital. The patient stated that she had had continuous contact with the dog for the previous year. The animal had a respiratory tract infection with cough, rhinorrhoea and fever for at least one week before its death. There was no history of exposure to other animals. Candida, coccidioidin, PPD and streptokinase-streptodornase skin tests were all negative, suggestive of an anergic state.

Physical examination revealed a cachectic female in respiratory distress. Blood pressure was $100 / 70$ $\mathrm{mmHg}$, temperature $39.4^{\circ} \mathrm{C}$, pulse $96 / \mathrm{min}$. Decreased breath sounds were noted on the right lung apex. There were coarse inspiratory rhonchi bilaterally. Chest X-ray showed persistence of the diffuse bilateral pulmonary infiltrates. The right 
chest tube was still in place. Haemoglobin was $9.6 \mathrm{~g} / \mathrm{dl}$, haematocrit $29.7 \%$, white blood count $16 \times 10^{9} / 1$ with $72 \%$ leucocytes, $22 \%$ stab cells and $4 \%$ eosinophils. Aspergillus titres, cold agglutinins, intermediate strength PPD, sputum acid-fast stains and cultures were negative. Blood and urine culture were negative. Gram stain of sputum from a Luken's aspirate showed many white blood cells, Grampositive cocci and Gram-negative coccobacilli. Cultures from the chest tube grew $S$. aureus and sputum cultures grew this organism plus $B$. bronchiseptica. The latter organism demonstrated the essential diagnostic characteristics outlined by Bemis, Greisen and Appel (1977a). As both organisms were sensitive to cephazolin, the patient was treated with this antibiotic for 10 days i.v. There was prompt defervescence and gradual resolution of the pneumonia. She was discharged 17 days after admission on an oral cephalosporin and advised to stay away from all animals.

\section{Discussion}

Little information is available in the medical literature concerning $B$. bronchiseptica. However, it is known that it is a common respiratory tract commensal of wild and domestic animals and has been responsible for outbreaks of fatal tracheobronchitis, pneumonia and septicaemia (Bemis et al., 1977b). B. bronchiseptica belongs to the order Eubacteriales and the family Brucellaceae. Other members of the genus include $B$. pertussis and parapertussis (Morse, 1973). All of these are Gramnegative coccobacilli. A good deal is known concerning the pathogenic characteristics of $B$. pertussis, particularly its ability to cause a severe inflammation of the tracheobronchial tree which often leads to secondary infection. Infrequently $B$. bronchiseptica has caused pertussis syndrome in American children (Brooksaler and Nelson, 1967).

$B$. bronchiseptica isolates have been recovered from the respiratory tracts of animal caretakers who were either asymptomatic or had a mild upper respiratory tract illness (Winnser, 1960). This implies that this organism can be transmitted from animal to man. The present patient is unlike pre- $\stackrel{\Phi}{\circledR}$ viously reported cases in that she had an underlying $C$. lympho-proliferative disease and that her dog was the probable source of her $\boldsymbol{B}$. bronchiseptica infec- $\stackrel{?}{\rightarrow}$ tion. The description of its infection and the course of its illness fits that of $B$. bronchiseptica infections $\frac{\bar{\sigma}}{\bar{N}}$ in canines.

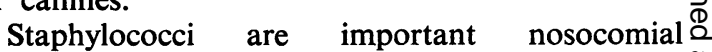
respiratory pathogens. Although most frequently क found as the sole infecting agent, mixed infections $\vec{\circ}$ due to $S$. aureus and other respiratory pathogens are well described (Musher and McKeune, 1977).

Perhaps $B$. bronchiseptica acts like $B$. pertussis, and predisposes the host to secondary infection. Infected patients with underlying neoplastic diseases $\frac{3}{3}$. need careful histories taken and a vigorous search $y$ for the causative organism. The authors feel that patients with lymphoproliferative disease and $\omega$ respiratory tract infections should be questioned $N$ about exposure to animals. If their history is positive, $\stackrel{\omega}{\circ}$ then $B$. bronchiseptica should be considered in the $\supset$ differential diagnosis of respiratory tract infections.

\section{References}

Bemis, D.A., Greisen, H.A. \& APPel, M.J.G. (1977a) Bacteriological variation among Bordetella bronchiseptiga isolates from dogs and other species. Journal of Clinit Microbiology, 5, 471.

Bemis, D.A., Gresien, H.A. \& APPel, M.J.G. (197 Pathogenesis of canine bordetellosis. Journal of Infectiouts Disease, 135, 753.

Brooksaler, F. \& Nelson, J.D. (1967) A reappraisal and report of 190 confirmed cases. American Journal of Diseases of Children, 14, 389.

Gardner, P., Griffin, W.B., Swartz, M.N. \& Kunz, L.J. $\stackrel{\text { \& }}{\varrho}$ (1970) Non-fermentative Gram-negative bacilli of noso- $\overrightarrow{\vec{\phi}}$ comial interest. American Journal of Medicine, 48, 735.

GoSh, H.K. \& TRANTER, J. (1979) Bordetella bronchiseptica infection in man: review and a case report. Journal of Clinical Pathology, 32, 546.

MORSE, S.I. (1973) The haemophilus-bordetella group. In: Microbiology. (Ed by Davis, B.D., Dulbecco, R., Eisen, $\frac{\partial}{3}$ H.N., Ginsberg, H.S., Wood, W.B. \& McCarty, M.), pp. 792-800. Harper and Row, New York.

Musher, D.M. \& MCKeUNE, S.O. (1977) Infections due to Staphylococcus aureus. Medicine. Baltimore, 56, 383.

WINNSER, J. (1960) A study of Bordetella bronchiseptica. Procedures in Animal Care Panel, 10, 87. 